\title{
Advanced Data Transfer Strategies for Overset Computational Methods
}

\author{
Eliot W. Quon ${ }^{\mathrm{a}, 1}$, Marilyn J. Smith ${ }^{\mathrm{a}, 2, *}$ \\ ${ }^{a}$ School of Aerospace Engineering, Georgia Institute of Technology, Atlanta, GA
}

\begin{abstract}
A data transfer strategy applicable to overset grid configurations has been developed that improves interpolation and extrapolation accuracy and eliminates orphan points. Traditional trilinear mappings based on interpolation stencils are replaced with a "cloud"-based algorithm which retains no dependence on grid connectivity. A variable number of donor points was sourced from a single grid in the vicinity of a receptor point, permitting consistent treatment of orphan points in the data transfer method. This cloud-based interpolation methodology demonstrates the ability to preserve flow-field features for configurations both with and without adequate mesh overlap. The approach eliminates problems associated with orphan points and reduces transient conservation errors by an order of magnitude.
\end{abstract}

Keywords: computational fluid dynamics, overset grids, data transfer, radial basis function interpolation

\section{Nomenclature}

$\alpha$ Radial basis interpolation coefficient

$\beta$ Polynomial interpolation coefficient

$\Delta s$ Isotropic grid spacing

\footnotetext{
* Corresponding author

Email address: marilyn.smith@ae.gatech.edu ( Marilyn J. Smith )

${ }^{1} \mathrm{Ph} . \mathrm{D}$. Candidate

${ }^{2}$ Professor
}

Preprint submitted to Computers \& Fluids

February 13, 2015 
${ }_{5} \Delta t$ Simulation time step size

$\Phi$ Radial basis kernel, $\Phi\left(\vec{x}, \vec{x}_{i}\right)=\phi\left(\left\|\vec{x}-\vec{x}_{i}\right\|_{2}\right)$

$\phi$ Radial basis function (RBF), $\phi(r)$

$h$ Hangar height

$M$ Mach number

10 $\quad N$ Number of interpolation source points

$n$ Normalized frequency, $f x_{r} / U_{\infty}$

$Q$ Number of polynomial coefficients

$r$ Radial (Euclidean) distance, $\|\vec{x}\|$

$s$ Interpolant to an unknown function

${ }_{15} V_{\infty}$ Free-stream velocity

$X$ Set of source data points

$x_{r}$ Reattachment point

$x_{s}$ Separation point

\section{Introduction}

Within the fluid mechanics community, many applications of interest involve predicting the unsteady behavior of configurations moving in multiple frames of reference. To facilitate the engineering analysis, an efficient means of handling the evolution of computational domains due to mesh motion, deformation, and/or grid adaptation is necessary. The state of the art in computational fluid 25 dynamics (CFD) is to use an overset or Chimera approach [1, 2, which applies overlapping grids for the time-accurate solution of unsteady problems and the modeling of complex geometries. This modular approach utilizes multiple body-fitted grids to model each moving component, in addition to one or more 
stationary background grids that model the remainder of the flow field. Overset grid systems permit interior grid boundaries to be placed arbitrarily so that different components may move freely relative to each other. The scheme has since been applied to both structured and unstructured grids for many engineering problems of interest $[3,4,5,6]$.

\subsection{The Overset Method}

An overset scheme requires that flow-field data be exchanged between pairs of overlapping meshes at each solver iteration to enable a solution on each component grid that is globally consistent. Points on non-solid interior boundaries requiring data exchange are known as fringe points. Additional effort is needed to obtain a solution because of the potentially complex domain interconnectivity between multiple overlapped grids. Moreover, since fringe points from neighboring grids are in general non-coincident, data transfer requires interpolation at each time step. Hole-cutting to remove points interior to solid boundaries, search operations to identify donor/receptor pairs, and calculation of interpolation weights are typically performed by additional software, such as PEGASUS 5 [7, PUNDIT [8], or Suggar++ [9]. The data transfer entails calculating a new solution at target locations known as receptors based on the solution from source points known as donors. On a Cartesian or structured mesh, the most efficient approach is to directly apply trilinear interpolation [10. The PEGASUS 5 grid preprocessor [7] employs this technique. A more general approach relies on isoparametric mappings with trilinear basis functions, applicable to both structured and unstructured grids. PUNDIT [8] and Suggar++ [1] both apply this technique.

Complications arise when acceptable donor points for interpolation cannot be found, giving rise to "orphan" points. This situation occurs if adjacent grids have insufficient overlap or if significant disparities in mesh spacing between grid levels exist. A point is considered to be an orphan when one or more of its donors is also a fringe point requiring an interpolated solution. An important consideration is that achieving higher-order spatial accuracy requires larger 
stencils. For example, an implicit sixth-order or explicit fourth-order scheme can require a five-point stencil which necessitates two levels of fringe points to maintain consistency with the interior of the computational domain [12, 13. When orphan points are present, solution fidelity may be lost because two levels of fringes cannot be resolved. Similarly, interpolation accuracy generally increases with overlap size because more points are available from which to perform the data transfer. The problem of orphan points is exacerbated by relative mesh motion which can increase the number of orphans and/or change their locations over time.

Solutions at orphan locations are typically estimated by an averaging procedure [14, 15, 6]. Two general mitigation approaches exist when orphan points are present. First, the grids may be redesigned to improve the quality of mesh intersections. In a recent application of an unstructured near-body methodology coupled to a Cartesian off-body solver, Abras and Hariharan [16 had to manually adjust the trim distance dictating the amount of overlap between near-body and off-body meshes. However, manual adjustments are not always possible, especially when considering complex geometries, and grid refinement can significantly increase cost. For example, a wing-store configuration studied by Power et al. 6] had $0.5 \%$ of all cells orphaned. Application of an adaptive mesh refinement procedure was able to eliminate all orphans but increased the total cell count by 10\%. Even if increased mesh sizes are acceptable, it may

so be difficult to guarantee that meshes in relative motion will be orphan-free for all time steps throughout the simulation. As an alternative, a dense interface grid may be added in the orphan region [17, 6]. These approaches require user intervention and added cost, either in engineering hours or computational time.

\subsection{Scattered Data Interpolation}

Scattered or cloud-based data techniques can provide a continuous mapping between arbitrarily structured data samples and remain decoupled from solver type (e.g., unsteady Reynolds-averaged Navier-Stokes, vorticity-velocity, or potential flow methodologies) and topology (Cartesian, structured, and unstruc- 
tured). More broadly described as kernel function interpolation, an interpolant is formed by a linear combination of nonlinear basis functions (kernels) to represent nonlinear functions. The approach is well established within other fields (e.g., computer graphics, digital elevation modeling, or optical design) but their application to CFD problems has been limited. This data transfer methodology has the potential to reduce or eliminate orphan points while increasing interpolation accuracy. Since donor points can be sourced from any location on any grid, the approach naturally precludes scenarios involving a lack of sufficient donor points.

When constructing an interpolant $(s)$ to an unknown function $(f)$ sampled from a set of scattered data points $(X)$, a solution is readily obtained when interpolation conditions

$$
s\left(x_{j}\right)=f_{j}, \quad x_{j} \in X,
$$

are independent of rigid (Euclidean) transformation [18. This is automatically the case when applying basis functions that depend only Euclidean distance $\left(r=\|x\|_{2}\right)[18$, i.e., radial basis functions (RBFs). The quality of the results is then sensitive to the location of kernel centers [19]. In the case of the centers coinciding with the locations at which the solution is known, an interpolant exists per the Mairhuber-Curtis theorem [20]. An additional consideration is that RBFs are by definition isotropic because the function has the same evaluation in all directions. However, fluid dynamics data are often discontinuous in nature. Therefore an alternative approach is to use not radial but elliptical bases to introduce data adaptivity into the data transfer algorithm. The development of anisotropic basis functions based on local solution gradients is described in Ref. 21, Rather than developing new basis functions, the present work focuses on applying established RBFs to the problem of overset data transfer.

Recommendations from a number of authors [22, 23, 20, have suggested that scattered data interpolation with RBFs is a general, accurate approach to transferring arbitrarily distributed data. These methods are especially attractive for overset data transfer because: 
1. They permit interpolation and extrapolation 24] based on arbitrarily clustered clouds of points in any dimensional space.

2. They have in general higher-order accuracy that can be increased by freely adding data points.

3. They are directly applicable to unstructured methodologies since the interpolant is decoupled from the computational mesh, eliminating requirements on the spatial structure of the sampled data.

4. They can be readily applied to the problem of solution transfer in overset methods since they do not require connectivity information.

For these reasons, an RBF approach is ideally suited for data transfer with orphan points since there are no overlap or connectivity requirements on the donor points. Therefore the same data transfer approach may be applied regardless of grid configuration.

An RBF is a univariate function of Euclidean distance from a chosen center $x_{c}$. Therefore the $\operatorname{RBF}(\phi)$ is related to its kernel function $(\Phi)$ as follows:

$$
\Phi\left(\mathbf{x}, \mathbf{x}_{c}\right)=\phi\left(\left\|\mathbf{x}-\mathbf{x}_{c}\right\|_{2}\right)=\phi(r) .
$$

An RBF interpolant based on the set of data samples $X$ has the following form:

$$
s_{f, X}(\mathbf{x})=\sum_{j=1}^{N} \alpha_{j} \Phi\left(\mathbf{x}, \mathbf{x}_{j}\right)+\sum_{k=1}^{Q} \beta_{k} p_{k}(x),
$$

where $s$ is the RBF interpolant of the function $f$ evaluated at an arbitrary location $x ; \alpha_{j}$ and $\beta_{j}$ are the interpolation coefficients to be determined; and $x_{j}$ are the RBF centers that coincide with the data sampling locations. Typically, $p_{k}$ is chosen to be a polynomial basis. An additional constraint is placed on the function $p_{k}$ to ensure solvability of the interpolation system [20]:

$$
\sum_{j=1}^{N} \alpha_{j} p_{k}\left(\mathbf{x}_{j}\right)=0 .
$$

Eqns. 3 and 4 are combined and solved as a linear system of equations:

$$
\left[\begin{array}{cc}
A_{\Phi, X} & P \\
P^{T} & 0
\end{array}\right]\left\{\begin{array}{l}
\alpha \\
\beta
\end{array}\right\}=\left\{\begin{array}{l}
f \\
0
\end{array}\right\},
$$


where the submatrices are defined as:

$$
\begin{gathered}
A_{\Phi, X}=\left(\Phi\left(\mathbf{x}_{j}, \mathbf{x}_{k}\right)\right) \in \mathbb{R}^{N \times N} \\
P=\left(p_{k}\left(x_{j}\right)\right) \in \mathbb{R}^{N \times Q} .
\end{gathered}
$$

Solution of Eqn. 5 produces an interpolant that depends on both spatial coordinates and the local solution field.

The potential challenges in applying RBF interpolation are widely understood [25, 26]. Effective interpolation requires a trade-off between the number of source points and numerical stability. Increasing the amount of source data generally increases accuracy but degrades the conditioning of the interpolation matrix (Eqn. 5). Even if the matrix inversion can be reliably performed, the cost of a direct evaluation using Gaussian elimination is $\mathcal{O}\left(N^{3}\right)$ [27, where $N$ is the number of source points. Therefore interpolation cost can quickly become a non-negligible portion of the overall simulation cost when transferring an entire overset solution field at once. A practical engineering solution is to employ a small subset of points (i.e., a cloud) within a neighborhood of the target point for each data transfer. Even if cost were not prohibitive, spurious fluctuations and solution degradation near clustered grid points may occur 24], further motivating a localized approach.

\section{Numerical Approach}

\subsection{Data Transfer}

The data transfer algorithms considered in the present work require accuracy comparable or greater than the solver order of accuracy (second order) for arbitrarily dense data. These algorithms have been applied to transfer discrete flow variable data between a single donor grid and a receptor grid. Focus is on two basis functions: the thin-plate spline (TPS) recommended in Refs. 28 and 29 for accuracy and robustness with both interpolation and extrapolation

$$
\phi(r)=r^{2} \log (r),
$$


and the compactly-supported Wendland $C^{2}$ (W2) basis function recommended in Refs. 30, 31, and 32

$$
\phi(r)= \begin{cases}(1-r)^{4}(4 r+1), & r \leq 1 \\ 0, & r>1 .\end{cases}
$$
to local subsets of data. The TPS is a global function defined for all $r$ and increases in value as $r \rightarrow \infty$. In contrast, the W2 function is a compact function which by definition decays to identically zero at a finite distance. For overset CFD problems of interest, application of global RBF interpolation using all data 170 samples is intractable since typical computational meshes have $\mathcal{O}\left(10^{6}\right)$ or greater 
points. Therefore in practice the data transfer will be limited to local subsets of points, motivating the use of compact basis functions. However, traditionally applied global RBFs that have demonstrated success for a variety of data 29] are not automatically precluded from the local approach. Therefore they have been investigated in this capacity as well.

The support radius for compact basis functions dictates the region of influence for each sample point, and consequently the number of sample points near the receptor that influence the solution at the receptor location. Increasing the support radius is therefore analogous to increasing the number of interpolation samples. In Eqn. 9, the radius $r$ has been normalized by the support radius so that the function is zero at a nominal distance of one. This normalization does not affect the inherent positive definite property of the basis function that ensures solvability of the interpolation problem [34. A constant value for the support radius does not need to be explicitly specified a priori as commonly reported in literature [34, 32 but instead can be permitted to vary depending on local point distribution 26, 33. Larger support radii will result in a smoother interpolated field [18, indicating that for fluid dynamics applications the choice of support radius is problem and grid dependent.

For varying mesh density as often encountered in complex fluid dynamics calculations, employing a fixed support radius can introduce problems by unnecessarily increasing smoothness in high point density regions or providing inadequate resolution in regions of low point density. Instead of explicitly specifying a support radius, sample points may be selected by fixing the number of nearest field points to include. Difficulties may arise for this approach both in selecting the number of points, and in selecting appropriate samples when available proximate field points are less than or greater than the desired number. As an alternative, the current work utilizes existing grid connectivity information to systematically select points near the receptor resulting in a cloud of more uniformly distributed data with an approximately constant number of donor 200 samples. The support radius is then not a free parameter but rather implicitly specified by normalizing the coordinates of the sample points to a unitary 
domain. This normalization is accomplished by translating the receptor and surrounding donor data points to the origin and scaling the coordinates of the interpolation sample points (i.e. the donor points) so that all points lie within $[-1,1]$ in each dimension. Data samples outside of the support radius are excluded. The same sampling and normalization approach is applied for both TPS and W2 interpolations.

Clouds of interpolation points are formed by first identifying the cell enclosing the receptor point. The cloud should include at minimum all nearest neighbors to the receptor point [34, which are the nodes of the enclosing computational cell. To verify the higher-order accuracy of this localized approach, interpolation of the two-dimensional Rastrigin function has been evaluated:

$$
f(x, y)=20+x^{2}+y^{2}-10 \cos (2 \pi x)-10 \cos (2 \pi y)
$$

This analytical function is of interest because it is both oscillatory and has a nonlinearly varying mean $\left[32\right.$. The solution on the $[-5.12,5.12]^{2}$ domain was transferred between non-coincident Cartesian grids of increasing mesh density, where mesh density is defined as the number of points in each direction. As illustrated in Fig. 1. interpolation errors may be reduced through mesh refinement or including more data samples. When interpolating with only the enclosing nodes (four donors in this case), RBF interpolation results in no appreciable improvement over bilinear interpolation; the observed differences in error were at most $\mathcal{O}\left(10^{-5}\right)$. If additional surrounding donor points are included, the convergence rate increases significantly. As the input mesh is refined, the highest observed error convergence rate is between fourth and fifth order (Table 1), comparable to the value of 4.30 for W2 interpolation of the same analytical function reported by Ref. 32. Both basis functions achieve at minimum second-order convergence as required, but W2 has higher-order convergence in this problem.

\subsection{Treatment of Orphan Points}

When applying a cloud-based interpolation method, the same approach to data transfer may be applied to configurations both with and without orphan 


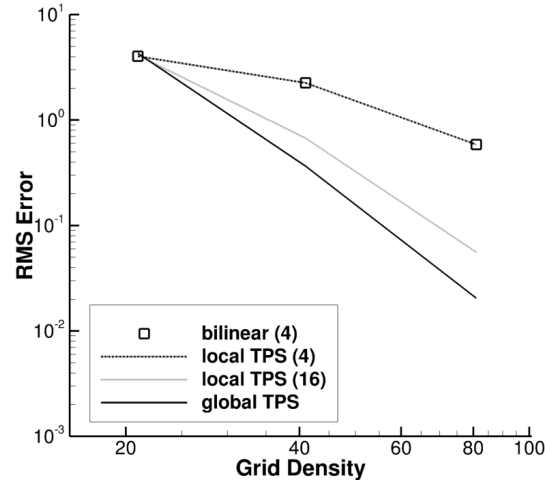

(a) Thin-plate spline

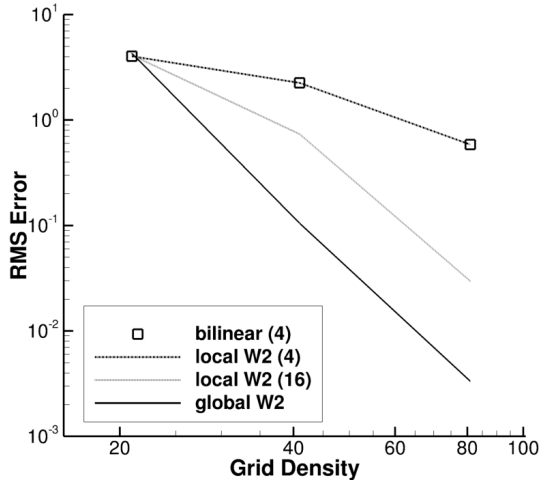

(b) Wendland $C^{2}$

Figure 1: Root-mean-square (RMS) interpolation error of an analytical test function; the number in parenthesis indicates the number of data samples included in the interpolation.

Table 1: Convergence rates (at $N=81$ ) for the Rastrigin function when applying localized and global interpolation approaches. $\infty$ indicates that all data samples were included for the global approach.

\begin{tabular}{lcc}
\hline \hline Method & Data Samples & Order \\
\hline Thin-plate spline & 4 & 1.98 \\
& 16 & 3.66 \\
& $\infty$ & 4.23 \\
\hline Wendland $C^{2}$ & 4 & 1.98 \\
& 16 & 4.72 \\
& $\infty$ & 5.07 \\
\hline \hline
\end{tabular}

225

points. Some examples of interpolation point clouds are illustrated in Fig. 2 as filled circles. In the current implementation, potential donor points that are also fringe points are removed from the interpolation cloud to permit explicit evaluation of the interpolant. Figure 2 outlines the enclosing cell whose nodes would have originally been used for linear interpolation. Additional neighboring nodes have been included to systematically increase the effective support radius. To maintain computational efficiency, expanding the cloud of points beyond a 
single level of surrounding nodes has not been considered. In the case of an orphaned fringe point where one or more donors are also orphans (Fig. 2p), the linear interpolant within the outlined cell cannot be explicitly formed. However, with the RBF data transfer algorithm, a valid solution is still obtained. Since the selected cloud of source points does not necessarily encompass the target point when considering orphans, the resulting data transfer may be an interpolation or extrapolation.

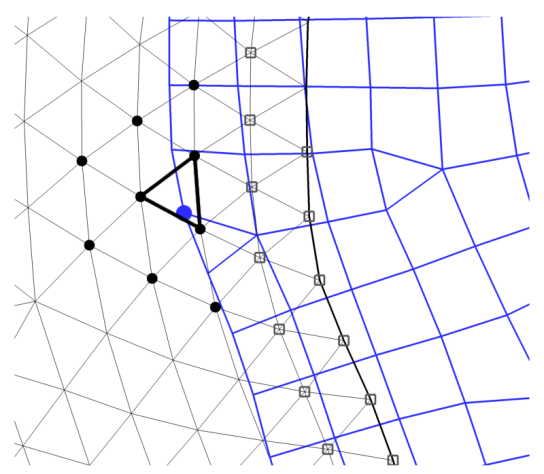

(a) Normal fringe point

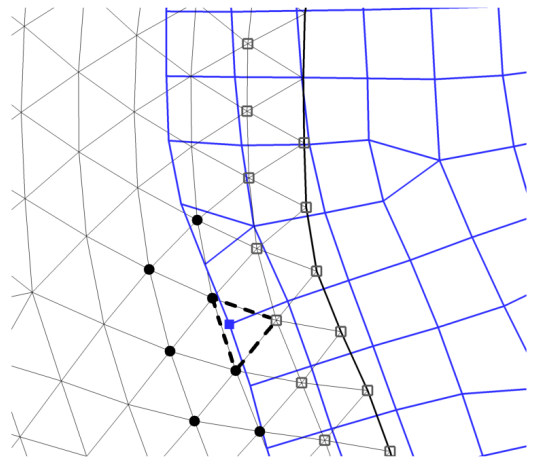

(b) Orphan fringe point

Figure 2: Clouds of source points in the vicinity of orphan points. Filled black circles denote selected donors while open square symbols indicate orphans. The cell enclosing the receptor point (filled blue circle) is outlined on the black grid.

Since there is no requirement that the receptor point be located within a particular cell (unlike standard mapping techniques) or that the donor points are connected to form a stencil as required for polynomial-based techniques, points may be arbitrarily included to compensate for a lack of resolution in the immediate vicinity of the receptor. The size of the cloud is allowed to vary in size to accommodate receptor points with insufficient donors. For instance, the number of non-fringe donors must be greater than or equal to the number of points comprising a single cell to maintain linear interpolation resolution (Fig. 1). If this condition is not met, then additional levels of neighboring nodes are added to the cloud until the minimum donor requirement is satisfied (Fig. 2(b)). 


\subsection{Computational Tools}

The baseline numerical analysis that was selected to develop and explore this overset concept is FUN3D [35, a fully unstructured, unsteady ReynoldsAveraged Navier-Stokes (URANS) solver developed primarily at NASA Langley Research Center. The present work used the incompressible path within FUN3D, which employs an artificial compressibility method to calculate the pressure and velocity fields satisfying the incompressible, viscous Navier-Stokes equations. Numerical schemes implemented in the code are spatially secondorder accurate with Roe upwinding and have second-order or higher temporal accuracy by applying multi-step backward difference formulas. For all cases in the present work, an optimized second-order temporal scheme with error controller 36] has been utilized. FUN3D includes overset mesh capabilities for accurate resolution of complex geometries and multiple frames of motion, for example in rotorcraft applications [37.

Two auxiliary codes are included to provide overset capabilities to FUN3D: Suggar++ (Structured, Unstructured Generalized overset Grid AssembleR) and DiRTlib (Donor Interpolation Receptor Transaction library) [14, 38. Suggar++ handles grid transformations and hole-cutting, donor-receptor identification, and calculation of interpolation weights; DiRTlib interfaces with the flow solver and uses the domain connectivity information generated by Suggar ++ to calculate interpolated values. For static meshes, Suggar ++ performs as a preprocessor to match donor and receptor points, after which interpolation weights are calculated based on donor locations only. For dynamic meshes, Suggar ++ is both a preprocessor and an iterative routine operating within FUN3D. At each solver time step and subiteration (for time-accurate simulations), DiRTlib retrieves the solutions at donor points and updates the solutions at fringe points. Pressure and velocity fields are updated through this process. To provide a cloud of source points for the scattered data technique, the standard donor search procedure has been modified to return not only the cell nodes encompassing the receptor node, but the neighbors of these cell nodes as well. Connectivity information is not required for the scattered data interpolation but has been utilized 
for convenience to avoid having to perform nearest neighbor searches.

\section{Evaluation Cases}

Two test cases have been considered in the present work. The first is the twodimensional simulation of an inviscid convecting vortex, a standard feature of many fluid dynamics problems. The second is the calculation of a ship airwake, which evaluates the methodology for a three-dimensional, turbulent flow.

\subsection{Inviscid Convecting Vortex}

A vortical disturbance is modeled in a form consistent with the FUN3D non-dimensionalization scheme [39]:

$$
\begin{aligned}
\frac{u}{U_{\infty}} & =1-\frac{\Gamma}{U_{\infty} R} \frac{z-z_{c}}{R} \exp \left(\frac{-r^{2}}{2}\right) \\
\frac{w}{U_{\infty}} & =\frac{\Gamma}{U_{\infty} R} \frac{x-x_{c}}{R} \exp \left(\frac{-r^{2}}{2}\right) \\
\frac{p-p_{\text {norm }}}{\rho_{\infty} U_{\infty}^{2}} & =1-\frac{\Gamma^{2}}{2 U_{\infty}^{2} R^{2}} \exp \left(-r^{2}\right) \\
r^{2} & =\frac{\left(x-x_{c}\right)^{2}+\left(z-z_{c}\right)^{2}}{R^{2}}
\end{aligned}
$$

for constant density $\rho=\rho_{\infty}$, free-stream reference velocity $U_{\infty}$, and $p_{\text {norm }}=$ $p_{\infty}-\rho_{\infty} U_{\infty}^{2}$. The vortex is centered at $\left(x_{c}, z_{c}\right)$ with reference length equal to

a nominal core radius $R$ of 1.0 and non-dimensional vortex strength $\Gamma / U_{\infty} R$ of 0.02. The pressure expression (Eqn. 13 is obtained by integrating $\frac{\partial p}{\partial r}=\frac{\rho_{\infty} u_{\theta}^{2}}{r}$ [40.

Overset simulations with trilinear interpolation performed data transfers with only the nodes of the cell enclosing the receptor point, which on average numbered between six and seven nodes. For this two-dimensional configuration, computational cells were primarily triangles that were extruded to form volumes for the three-dimensional flow solver. These triangles became six-noded pentahedra (while quadrilaterals became eight-noded hexahedra), hence trilinear rather than bilinear interpolation was performed. The number of donor points was fixed for the linear approach, regardless of whether orphan points 
were present. In comparison, the RBF interpolations were based upon clouds of 23-24 nodes on average, nearly a fourfold increase. This indicates that most triangles are connected to nine other nodes on average for a total of 12 nodes on a two-dimensional plane. In the presence of orphan points, the number of available donors decreased by 19-38\%, and the minimum number of points per cloud was 10. All donor statistics are summarized in Table 3

Unstructured test configurations with and without orphan points were created from the meshes depicted in Fig. 3. A configuration with orphan points was created by resizing the background grid (Fig. 3(a) to have an approximately 20\% larger cutout region. This grid was overset with a patch grid that was not resized (Fig. 3(b) , thus reducing the amount of overlap between grids. Baseline, fine, and coarse meshes were considered corresponding to isotropic cell sizes of $\Delta s=0.2,0.1$, and 0.4 , respectively. In the baseline case $(\Delta s=0.2)$, the reduced overlap region transformed over half of the fringe points $(2,036)$ into 1,152 orphans points. These orphan points are illustrated by the symbols in Fig. 3(c) Since these orphan points cannot be adequately resolved with the original overset interpolation paradigm, an effective gap in the computational grid is formed. On the coarse mesh $(\Delta s=0.4)$, the number of orphan points was an order of magnitude larger than the number of calculable fringe points.

320 This represents an extremely unlikely, but very rigorous evaluation case. All grids studied in the present section are summarized in Table 2

Results were computed with a time step of $\Delta t=0.005$. A temporal study determined that this step size is sufficiently small to permit analysis of spatial errors [21]. Calculations on baseline grids with a spacing of $\Delta s=0.2$ resulted in the predicted flow fields illustrated by Fig. 4. The orphan-free overset solution (Fig. 4(b) preserved the same qualitative features as the single-grid case (Fig. 4(a) , which exhibited minor dissipation due to the relative coarseness of the mesh. If orphan points are present in the grid configuration, the traditional trilinear approach is not well defined since the flow field is not known at one or more constituent nodes of the donor cell. In this case, values at receptor points are determined by averaging rather than interpolation [14. This 
Table 2: Unstructured grids used in the vortex convection study with orphan points

\begin{tabular}{lcccc}
\hline \hline Grids & Grid Spacing $(\Delta s)$ & Nodes & Fringes & Orphans \\
\hline Vortex, single grid & 0.4 & 8,528 & - & - \\
& 0.36 & 10,350 & - & - \\
& 0.2 & 33,646 & - & - \\
& 0.1 & 133,640 & - & - \\
\hline Vortex, overset & 0.4 & 11,128 & 1,154 & 0 \\
& 0.2 & 40,944 & 1,894 & 0 \\
& 0.1 & 162,808 & 4,074 & 0 \\
\hline Vortex, overset & 0.4 & 9,352 & 158 & 1,040 \\
with orphan points & 0.2 & 33,256 & 884 & 1,152 \\
& 0.1 & 129,818 & 3,394 & 1,008 \\
\hline \hline
\end{tabular}

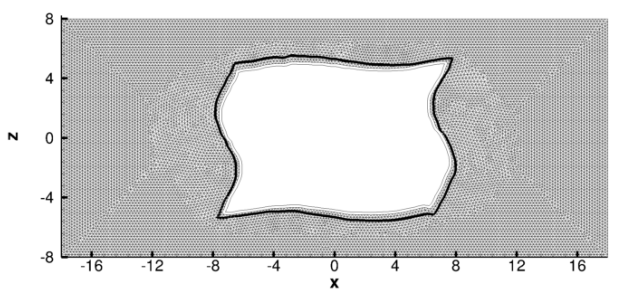

(a) Unstructured background grid with irregular cutout

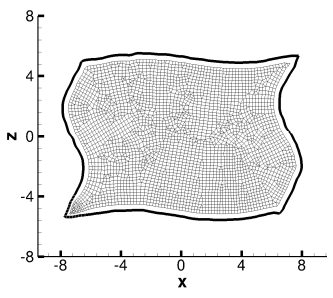

(b) Irregular patch grid

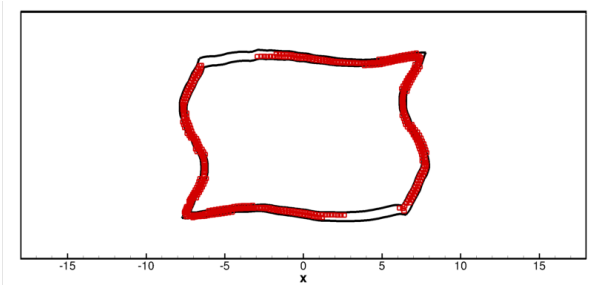

(c) Orphan points

Figure 3: Overset grid systems for the convecting vortex calculation (baseline grids, $\Delta s=0.2$ ). The outlined region denotes the extent of the patch grid. Orphan points are indicated by red square symbols. 
Table 3: Donor statistics for the vortex convection study.

\begin{tabular}{ccccc}
\hline \hline Overset Configuration & Mesh Spacing & Avg. & Min. & Max. \\
\hline Trilinear interpolation & 0.4 & 6.71 & 6 & 8 \\
& 0.2 & 6.84 & 6 & 8 \\
& 0.1 & 6.78 & 6 & 8 \\
\hline Trilinear interpolation & 0.4 & 6.56 & 6 & 8 \\
with orphans & 0.2 & 6.84 & 6 & 8 \\
& 0.1 & 6.82 & 6 & 8 \\
\hline RBF interpolation & 0.4 & 22.85 & 18 & 28 \\
& 0.2 & 24.02 & 18 & 28 \\
& 0.1 & 24.06 & 18 & 32 \\
\hline RBF interpolation & 0.4 & 14.11 & 10 & 24 \\
with orphans & 0.2 & 16.23 & 10 & 26 \\
& 0.1 & 19.59 & 10 & 26 \\
\hline \hline
\end{tabular}

procedure tended to dissipate the vortex structure after passage through the upstream and downstream overset boundaries (Fig. 4(c)). In comparison, the scattered data transfer approach utilizing RBFs eliminates the uncertainty at orphan locations arising from any averaging procedure. The RBF algorithm systematically includes additional data in the interpolation to maintain resolution at orphan locations.

The root-mean-square (RMS) errors between the computation and the exact solution are tabulated in Table 4 for the baseline case after the vortex has convected in and out of the overset patch region. As expected for the configurations without orphan points, the errors in each solution variable are the same order of magnitude, and differences between the single and overset grid configuration are $\mathcal{O}\left(10^{-5}\right)$ or less and are therefore negligible. In the overset cases, the error introduced by interpolation is an order of magnitude less than the discretization error (observed in the single-grid case). For all configurations with and without 
orphan points, the RBF algorithm consistently results in lower error than the traditional calculation with trilinear interpolation.

When orphan points were introduced into the computational mesh, the velocity errors in the calculation with trilinear interpolation increased by a factor of three to four over the orphan-free case. The largest change in error occurs for pressure, which increased by an order of magnitude (Table 4). Application of either TPS or W2 interpolation mitigated the RMS error and successfully preserved the structure of the vortex (Fig. 4(d)]. Compared to the trilinear interpolated solution, the errors in $u$ and $w$ were reduced by $59 \%$ and $45 \%$, respectively, and the error in interpolated pressure reduced by an order of magnitude. In comparison with an orphan-free configuration, the vortex structure is slightly skewed with the deformation approximating the shape of overset gap (Fig. 4(d) . Results from applying the W2 basis were visually indistinguishable from the TPS approach, and is not shown. Differences between the two functions are at most $3 \%$ and an order of magnitude smaller than the observed error (Table 4 .

To reduce discretization error and permit more accurate evaluations of overset interpolation error, refined versions of the grids in Fig. 3 (with $\Delta s=0.1$ ) were created. RMS errors for the fine grid case are tabulated in Table 5 . In 365 comparison with the results in Table 4 the orphan-free errors have decreased by a factor of four in all cases. This indicates that the overall spatial errors are exhibiting second-order convergence as expected from the spatial scheme. Despite having a more refined mesh, the presence of orphan points resulted in 25-73\% higher error compared to the orphan-free overset solution when applying trilinear interpolation. In comparison, the TPS and W2 overset solutions only exhibited increases in error of up to $11 \%$ and $18 \%$ respectively. When applying either scattered data transfer technique, spatial errors are reduced by over $30 \%$ in streamwise velocity and pressure, and by $16 \%$ in normal velocity. The TPS and W2 solutions with orphans are within $5 \%$ of the orphan-free trilinear overset solution in terms of streamwise velocity and normal velocity, while the difference in pressure error is an order of magnitude smaller than the velocity 


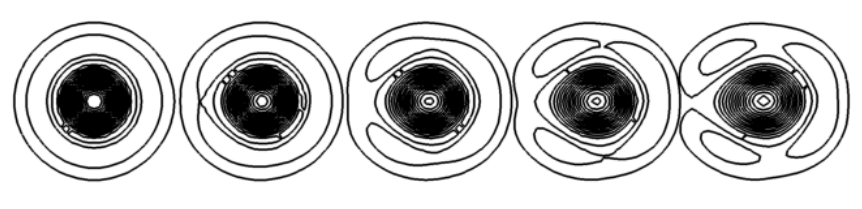

(a) Single Grid

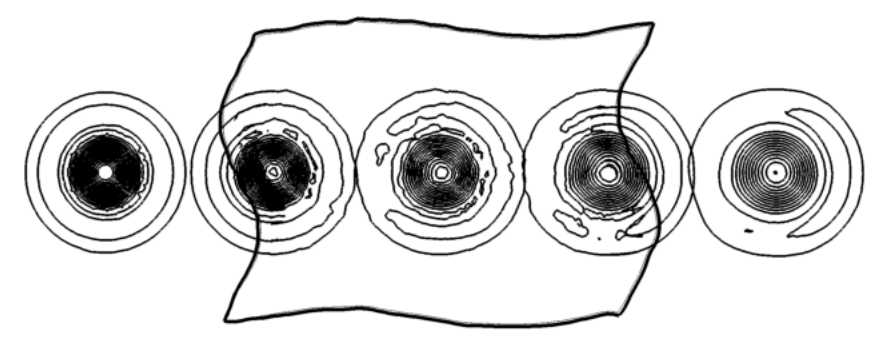

(b) Overset, no orphans, trilinear interpolation

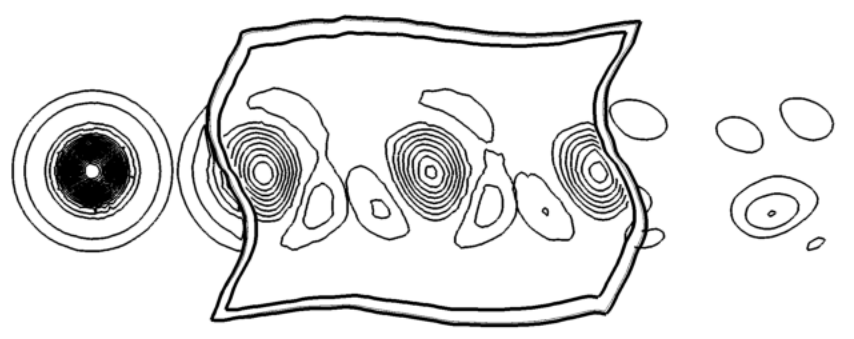

(c) Overset, with orphans and trilinear interpolation

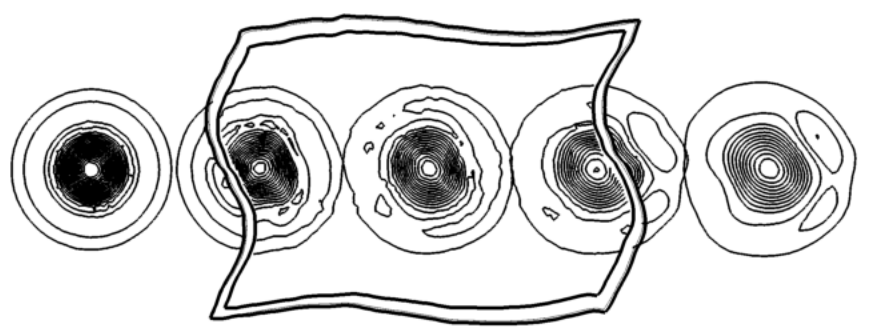

(d) Overset, with orphans and RBF interpolation

Figure 4: A comparison of the vorticity field for a single-grid case, an overset case without orphans, and two overset cases with orphans. The solution at five instances in time have been superimposed on the same image. All calculations were performed on the baseline mesh $(\Delta s=0.2)$. 
Table 4: Root-mean-square errors for overset solutions with and without orphans compared to the single-grid case (baseline meshes, $\Delta s=0.2$ ).

\begin{tabular}{lccc}
\hline \hline Test Case & $u$ error & $w$ error & $p$ error \\
\hline Single Grid & $2.2 \times 10^{-4}$ & $2.7 \times 10^{-4}$ & $3.9 \times 10^{-6}$ \\
Overset, trilinear & $2.4 \times 10^{-4}$ & $3.0 \times 10^{-4}$ & $4.4 \times 10^{-6}$ \\
Overset, TPS & $2.3 \times 10^{-4}$ & $2.9 \times 10^{-4}$ & $4.2 \times 10^{-6}$ \\
Overset, W2 & $2.2 \times 10^{-4}$ & $2.8 \times 10^{-4}$ & $4.1 \times 10^{-6}$ \\
\hline Overset with orphans, trilinear & $9.7 \times 10^{-4}$ & $8.9 \times 10^{-4}$ & $7.4 \times 10^{-5}$ \\
Overset with orphans, TPS & $4.0 \times 10^{-4}$ & $4.9 \times 10^{-4}$ & $6.6 \times 10^{-6}$ \\
Overset with orphans, W2 & $4.0 \times 10^{-4}$ & $4.9 \times 10^{-4}$ & $6.8 \times 10^{-6}$ \\
\hline \hline
\end{tabular}

Table 5: Root-mean-square errors for overset solutions with and without orphans compared to the single-grid case (fine mesh, $\Delta s=0.1$ ).

\begin{tabular}{lccc}
\hline \hline Test Case & $u$ error & $w$ error & $p$ error \\
\hline Single Grid & $4.9 \times 10^{-5}$ & $7.2 \times 10^{-5}$ & $1.0 \times 10^{-6}$ \\
Overset, trilinear & $5.8 \times 10^{-5}$ & $8.0 \times 10^{-5}$ & $1.1 \times 10^{-6}$ \\
Overset, TPS & $5.4 \times 10^{-5}$ & $7.7 \times 10^{-5}$ & $1.1 \times 10^{-6}$ \\
Overset, W2 & $5.2 \times 10^{-5}$ & $7.6 \times 10^{-5}$ & $1.1 \times 10^{-6}$ \\
\hline Overset with orphans, trilinear & $8.6 \times 10^{-5}$ & $1.0 \times 10^{-4}$ & $1.9 \times 10^{-6}$ \\
Overset with orphans, TPS & $6.0 \times 10^{-5}$ & $8.4 \times 10^{-5}$ & $1.2 \times 10^{-6}$ \\
Overset with orphans, W2 & $5.9 \times 10^{-5}$ & $8.4 \times 10^{-5}$ & $1.3 \times 10^{-6}$ \\
\hline \hline
\end{tabular}


Table 6: Root-mean-square errors for overset solutions with and without orphans compared to the single grid case (coarse mesh, $\Delta s=0.4$ ).

\begin{tabular}{lccc}
\hline \hline Test Case & $u$ error & $w$ error & $p$ error \\
\hline Single Grid & $5.88 \times 10^{-4}$ & $6.42 \times 10^{-4}$ & $1.03 \times 10^{-5}$ \\
Single Grid $(\Delta s=0.36)$ & $5.33 \times 10^{-4}$ & $5.47 \times 10^{-4}$ & $8.46 \times 10^{-6}$ \\
Overset & $5.70 \times 10^{-4}$ & $6.45 \times 10^{-4}$ & $1.02 \times 10^{-5}$ \\
Overset, TPS & $5.43 \times 10^{-4}$ & $6.32 \times 10^{-4}$ & $9.95 \times 10^{-6}$ \\
Overset, W2 & $5.34 \times 10^{-4}$ & $6.29 \times 10^{-4}$ & $1.02 \times 10^{-5}$ \\
\hline Overset with orphans & $1.05 \times 10^{-3}$ & $1.04 \times 10^{-3}$ & $4.34 \times 10^{-5}$ \\
Overset with orphans, TPS & $8.82 \times 10^{-4}$ & $9.50 \times 10^{-4}$ & $1.11 \times 10^{-4}$ \\
Overset with orphans, W2 & $8.92 \times 10^{-4}$ & $9.52 \times 10^{-4}$ & $4.27 \times 10^{-4}$ \\
\hline \hline
\end{tabular}

errors.

Alternatively, coarse grids $(\Delta s \approx 0.4)$ were evaluated to study the accumulation of interpolation errors with decreasing spatial resolution. Cell sizes in this grid configuration are larger than recommended for CFD simulations. The error in all three solution variables was actually comparable to or lower than the single-grid configuration. This is not surprising when considering that the overset configuration had $30 \%$ more nodes, thus provided sufficient additional spatial resolution to reduce errors. To enable more accurate comparisons between the single and overset grid configurations, the single-grid spacing was slightly reduced from $\Delta s=0.4$ to 0.36 to more closely match the number of nodes (Table 2 in both configurations. When orphan points are present in the grid, the advanced data transfer results demonstrate improvement over the linear data transfer with up to $16 \%$ and $9 \%$ error reduction in streamwise and normal velocities. Compared to the other grid configurations tested, the effectiveness of the scattered data interpolation is limited by the quality of the sampled data. This is especially apparent when considering the pressure errors. The pressure field on the coarse grid $(\Delta s \approx 0.4)$ exhibits only very small variations compared to the other variables $\left(\mathcal{O}\left(10^{-4}\right)\right)$ due to the $\Gamma^{2}$ term in 
the vortex definition (Eqn. 13). Therefore it was sensitive to any higher-order interpolation errors such as over- or under-shoot, illustrated by the fact that pressure errors are larger with either RBF approach than the linear approach when orphan points are present. For this case, the W2 pressure solution has nearly four times the error of the TPS solution. Accumulation of interpolation errors was also observed in Ref. 21 for a rotor blade solution repeatedly transferred between fine and coarse meshes, which similarly demonstrated that the TPS solution tends to be more stable than the W2 solution. This behavior only occurs when the solution significantly deteriorates due to both lack of grid resolution and the presence of orphan points and represents a situation that is not likely to be encountered in practice.

To obtain an accurate estimate of data transfer effectiveness, the spatial errors have been separated from temporal error. A temporal convergence study (with $\Delta t=0.005,0.01,0.02, \ldots, 0.32$ ) was performed to isolate the spatial error as $\Delta t \rightarrow 0$, and series convergence was accelerated with the Richardson extrapolation technique 41. Data presented in Tables $4 \sqrt{6}$ correspond to $\Delta t=0.005$, the finest time step examined. Based on these extrapolated errors, the overset interpolation error was determined by subtracting the single-grid spatial error from the overset spatial errors. The resulting isolated overset errors are plotted in Fig. 5 In general, configurations both with and without orphan points tend to converge at the finest grid level tested for the linear and TPS overset approaches, and the RBF interpolation approaches consistently outperformed trilinear interpolation. In the orphan-free configurations, consistent improvements from applying the RBF approach were observed in the velocity variables. At the baseline grid level, isolated overset errors in streamwise velocity were reduced up to $71 \%$ compared to trilinear interpolation when applying TPS interpolation and an order of magnitude when applying W2 interpolation. Improvements of up to $39 \%$ and $52 \%$ were observed in normal velocity for TPS and W2, respectively. For configurations with orphan points, the TPS and W2 results were typically indistinguishable with approximately half an order of mag425 nitude reduction in overset error at the baseline and fine grid levels compared to 


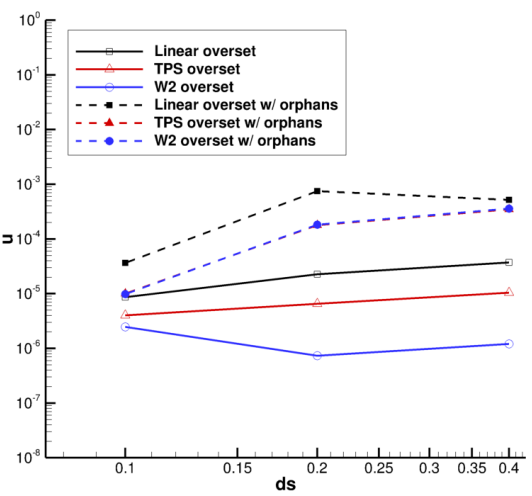

(a) Streamwise Velocity

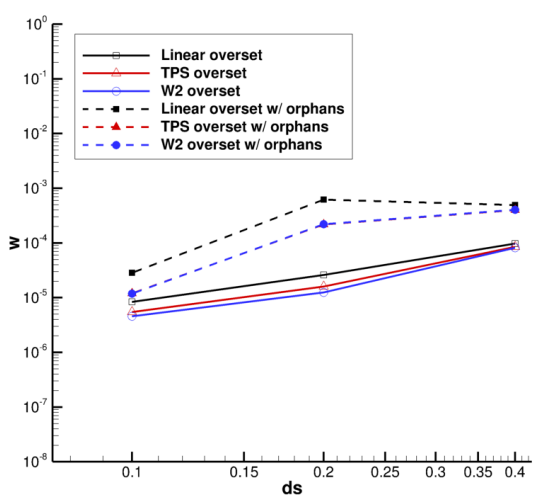

(b) Normal Velocity

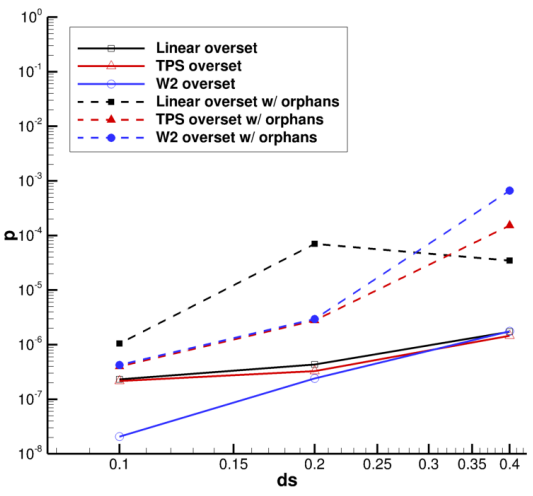

(c) Pressure

Figure 5: Richardson extrapolated overset errors for various data transfer strategies on configurations with and without orphans.

the trilinear approach. Errors were slightly higher at the baseline grid spacing $(\Delta s=0.2)$ than the coarsest grid $(\Delta s=0.4)$ because there were $11 \%$ more orphan points in the baseline configuration (Table 2). The isolated pressure error on the coarse $\Delta s=0.4$ configuration again illustrates the possibility for the higher-order interpolation to introduce additional error when the quality of the sampled data is low.

The final part of the convecting vortex analysis focuses on the extent to which the conservation laws of fluid dynamics are satisfied when applying the various interpolation methods. This may be evaluated by considering the net 
mass and momentum fluxes in and out of the computational domain. Any deviation from zero is considered conservation error. These conservation errors were calculated from instantaneous solutions for linear and RBF interpolation methods on the baseline grid with orphans. Romberg integration was applied to estimate the numerical fluxes in the limit of zero grid spacing. For $N=2^{k}+1$

equally spaced points where $k$ is a positive integer, the Romberg algorithm performs $k$ iterations to remove error terms up to $\mathcal{O}\left(1 / N^{2 k}\right)$ [42. To initiate this procedure, the boundary solutions are collocated to provide a number of equally spaced points equal to $2^{k}+1$. The shortest integration path lies along the upstream and downstream boundaries. These boundaries have the fewest points and will have the highest theoretical error. On these boundaries, 81 points (on the baseline mesh) are interpolated to 129 locations (for $k=7$ ) and results in an integration error $\sim \mathcal{O}\left(\Delta s^{2 k}\right) \approx 10^{-10}$.

The conservation errors in mass, $x$-momentum, and $z$-momentum are plotted in Fig. 6 for the baseline grid level. For all three quantities, the error on the single grid is of the order $10^{-7}$, which is greater than the error in the numerical integration and therefore significant. Errors are introduced in the overset simulations when the vortex is in the vicinity of the overset interfaces at $x \approx-6$ and $x \approx 6$. These errors appear as local maxima in the computed net flux, with double peaks appearing prominently on the coarser mesh at $x \approx-9,-5,6$, and 9 (Figs. 6, left column). The peaks correspond to the passage of the front and rear of the vortex core where swirl velocity and velocity gradients are at a maximum. After the vortex passage, the initial conservation error level may not be immediately recovered because the maximum number of subiterations was fixed for all simulations. Application of advanced data transfer techniques reduced maximum conservation errors by approximately $80 \%-90 \%$ each time the vortex passed through an overset boundary.

The mass conservation error returns to the approximate unperturbed level at $x \approx-4$ after the vortex core has crossed from the background onto the overset patch grid. After the vortex moves from the patch back to the background grid 465 through the second set of fringe points (beginning at $x \approx 4$ ), the accumulated 

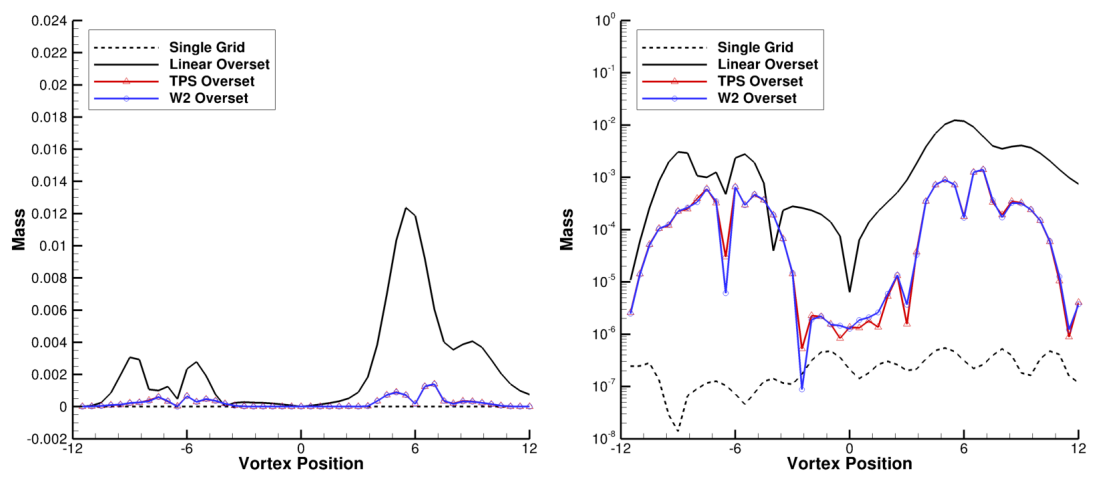

(a) Mass
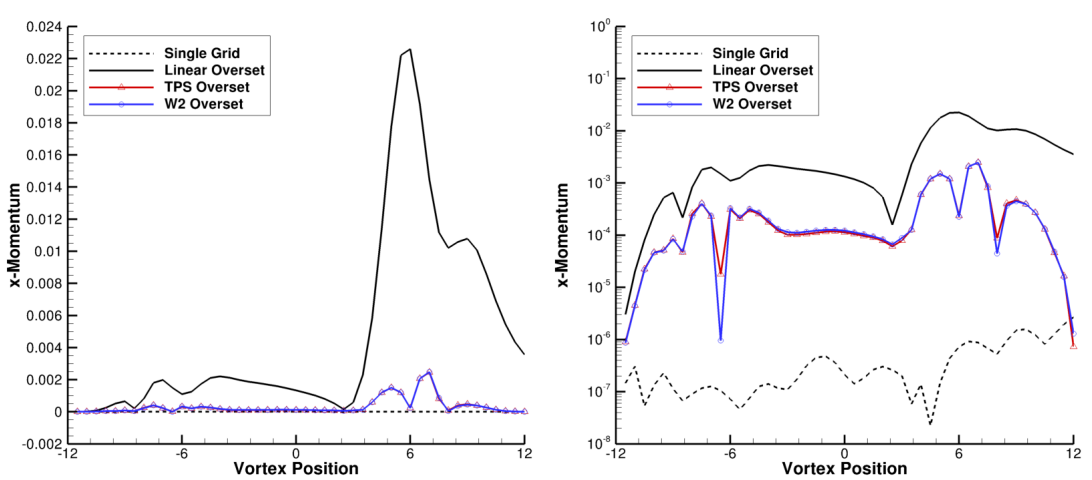

(b) $x$-Momentum
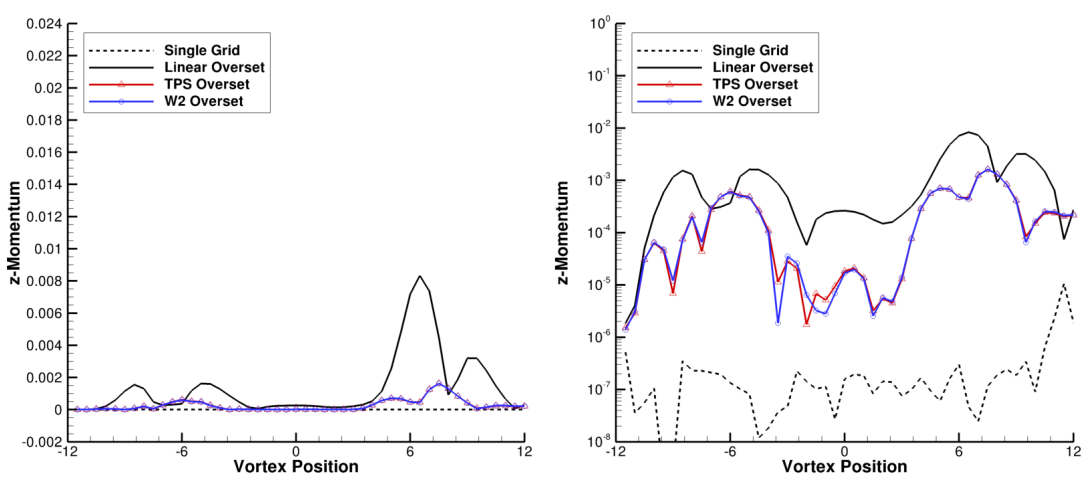

(c) $z$-Momentum

Figure 6: Calculated net flux through the outer boundary of the computational domain for the inviscid convecting vortex test case. The left column presents results on a linear scale to elucidate the effect of vortex passage through overset boundaries with orphans. The right column presents the same errors on a logarithmic scale. 
error is consistently higher than the passage through the first set of fringes. While the fluxes in both linear and RBF overset simulations display the same trends over time or as a function of vortex position, applying a RBF interpolation technique does not simply scale the errors. For instance, the large increase

level (Fig. 6, right column) when applying trilinear interpolation. In addition, the mass and $x$-momentum fluxes have not returned to initial levels even at the end of the simulation (Figs. 6a and b). For more complex aerodynamics, the accumulation of overset conservation errors may be more significant and require additional spatial and temporal refinement than in current engineering practice.

The cost of simulating each of the vortex cases on a single processor is summarized in Table 7. For all cases, the cost of applying either RBF was within $2.5 \%$ of each other. For cases without orphans, the cost increase associated with applying RBF interpolation is negligible as illustrated in Fig. 7 At the coarsest grid level, the cost increase over trilinear interpolation is $17 \%$ which corresponded to an increase in average wallclock time of less than 0.1 seconds per iteration. On larger meshes, the increase in average wallclock time is less than 0.3 seconds per iteration, corresponding to less than $3 \%$ increase in cost over the linear overset approach.

When orphan points exist in the grid configuration, the linear overset approach applies an averaging procedure whereas the RBF approach interpolates all fringe points in the same manner. The increase in cost of the RBF approaches comes from an approximate fourfold increase in the number of subiterations required ("Avg. subiters." in Table 7) to reduce the temporal error by an order of magnitude, which is offset by the number of donor points decreasing by approximately 20-40\% (Table 3). The decrease in average donors per fringe point is due to orphaned points being rejected as suitable donors in the data transfer. As mesh density increases, the number of suitable donor points also increases (e.g. from 14 to 20 donors on average, Table 3 so that the number of donors 495 approaches the same number as the orphan-free simulations. In general, the cost scales approximately as $\mathcal{O}\left(N^{2}\right)$ where $N$ is the number of fringe points 
Table 7: Cost in terms of averaged wallclock time per step for the test cases and methods presented.

\begin{tabular}{lcccccc}
\hline \hline Case & Method & $\begin{array}{c}\text { Grid } \\
\text { spacing }\end{array}$ & Fringes & $\begin{array}{c}\text { Avg. } \\
\text { subiters. }\end{array}$ & $\begin{array}{c}\text { Cost / } \\
\text { step [s] }\end{array}$ & $\begin{array}{c}\text { \% incr. } \\
\text { in cost }\end{array}$ \\
\hline Vortex & Linear & 0.4 & 1,154 & 8.08 & 0.4167 & - \\
Vortex & TPS & 0.4 & 1,154 & 8.29 & 0.4860 & $16.6 \%$ \\
Vortex & W2 & 0.4 & 1,154 & 8.32 & 0.4789 & $14.9 \%$ \\
Vortex & Linear & 0.2 & 1,894 & 8.87 & 2.1718 & - \\
Vortex & TPS & 0.2 & 1,894 & 9.02 & 2.3416 & $7.8 \%$ \\
Vortex & W2 & 0.2 & 1,894 & 9.02 & 2.2868 & $5.3 \%$ \\
Vortex & Linear & 0.1 & 4,074 & 9.80 & 10.3831 & - \\
Vortex & TPS & 0.1 & 4,074 & 9.84 & 10.6672 & $2.7 \%$ \\
Vortex & W2 & 0.1 & 4,074 & 9.79 & 10.6085 & $2.2 \%$ \\
\hline Vortex w/ orphans & Linear & 0.4 & 158 & 16.3 & 0.7830 & - \\
Vortex w/ orphans & TPS & 0.4 & 1,198 & 39.7 & 2.1038 & $168.7 \%$ \\
Vortex w/ orphans & W2 & 0.4 & 1,198 & 39.6 & 2.1021 & $168.5 \%$ \\
Vortex w/ orphans & Linear & 0.2 & 884 & 36.2 & 4.6648 & - \\
Vortex w/ orphans & TPS & 0.2 & 2,036 & 37.1 & 5.2125 & $11.7 \%$ \\
Vortex w/ orphans & W2 & 0.2 & 2,036 & 36.0 & 5.1956 & $11.4 \%$ \\
Vortex w/ orphans & Linear & 0.1 & 3,394 & 38.6 & 17.6546 & - \\
Vortex w/ orphans & TPS & 0.1 & 4,402 & 38.9 & 20.1944 & $14.4 \%$ \\
Vortex w/ orphans & W2 & 0.1 & 4,402 & 38.9 & 20.4251 & $15.7 \%$ \\
\hline \hline
\end{tabular}

(Fig. 7). This quadratic relationship is related to the cost of multiplying the inverse of the left-hand-side matrix in Eqn. 5 to evaluate the interpolation coefficients. While the cost of the matrix inversion is $\mathcal{O}\left(N^{3}\right)$, this is incurred in a 500 preprocessing step rather than at run time. 


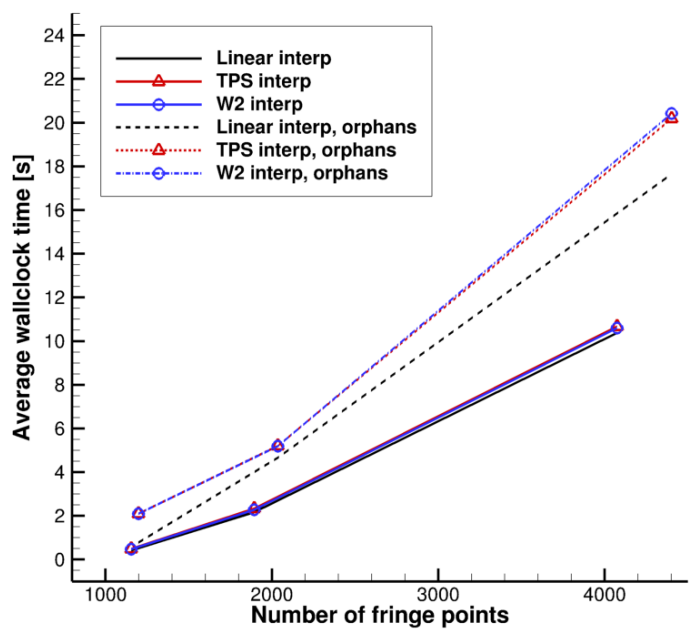

Figure 7: Average wallclock time required per solver iteration as a function of the number of overset fringe points.

\subsection{Ship Airwake Simulations}

The advanced overset methodology has been further evaluated on the turbulent three-dimensional airwake of a model frigate (Fig. 8). An overset approach has been applied to study a naval ship airwake, which can provide flexibility in modeling the ship under different operating conditions as well as motions. In the present study, a 1/50th-scale model at $70.6 \mathrm{ft} / \mathrm{s}$ headwind conditions has been simulated at a computational time step equal to $0.0005 \mathrm{~s}$ per step. This step size follows previous investigations of the same geometry in which highly unsteady flow was observed in the deck region [44]. The ship is modeled with viscous surfaces and the far field is modeled with a Riemann invariant condition. The sea boundary is considered to be part of the far field, neglecting the moving interface between the air and water. Turbulent quantities were calculated with a hybrid RANS/Large Eddy Simulation technique implemented in FUN3D that captures the complex physics at large spatial scales on the order of the mesh cell size, and models only the behavior of scales smaller than the cell size [45].

A single grid configuration was created for reference as a best possible solution to compare with the overset solutions. The overset configuration included 


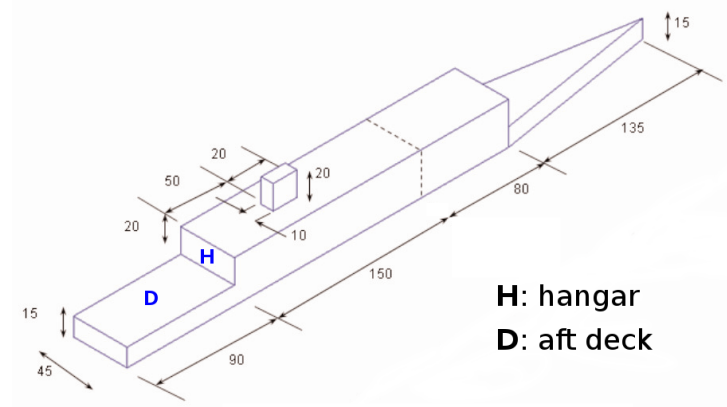

Figure 8: SFS ship geometry with dimensions in feet, from Ref. 43

two grids, a near-body ship grid and a background grid. The same ship surface grid with $y^{+}<1$ was included in both grid models. All grids were centered on ${ }_{520}$ the ship center and had far-field boundaries extending outward 4.5 ship lengths $\left(L_{s}\right)$ in the streamwise and lateral directions, and $0.75 L_{s}$ normal to the sea plane. The near-body grid in the overset configuration extended $0.75 L_{s}$ and $0.5 L_{s}$ ship lengths in the outward and normal directions from ship center. To maintain similarity between the single and overset configurations, the point distribution at the outer boundary of the near-body overset grid was enforced in the same region within the single-grid configuration. The final single and overset grids contained 2.7 and 3.0 million nodes, respectively. The overset configuration contained 31,982 fringe points on overset boundaries, exclusive of interior fringe points. Remaining additional nodes are accounted for by the overlapping portions of the fluid domain solved by both grids.

Analysis focuses on the aft ship deck region (indicated in Fig. 8 because it is critical to naval aircraft operations and because the flow can be related to backward-facing step physics. Velocity spectra were calculated from solutions sampled above the center of the deck at the height of the aircraft hangar. A dominant frequency of approximately $7.8 \mathrm{~Hz}$ was found from a Fourier analysis in both streamwise and normal velocity components for all configurations (Fig. 91. Flow-field averaging in all cases was therefore performed over a period of 0.128 seconds (corresponding to $7.8 \mathrm{~Hz}$ ), equal to the time required for the 
flow to traverse the length of the ship once or the deck five times. This period includes 256 data samples taken at 0.0005 second intervals. The sampling interval is equal to the computational time step size.

The flow conditions in this simulation correspond to a Reynolds number based on hangar height of $1.82 \times 10^{5}$ and the flow has been assumed to be fully turbulent. At this Reynolds number, the maximum contribution to energy in the

${ }_{545}$ flow is expected to occur at a normalized frequency of $n \equiv \frac{f x_{r}}{U_{\infty}} \approx 0.1$ [46, where $x_{r}$ is the mean reattachment length, the location at which the flow reattaches on the deck behind the circulating region. This low frequency behavior is indicative of an unsteady "flapping" of the shear layer throughout the separated region of the field 47. For the current test case, the detected frequency of $7.8 \mathrm{~Hz}$ equates to $n=0.113$. Computations agree with experimental results to within $13 \%$ and the differences are attributed to the added geometric complexity in the SFS2 model.

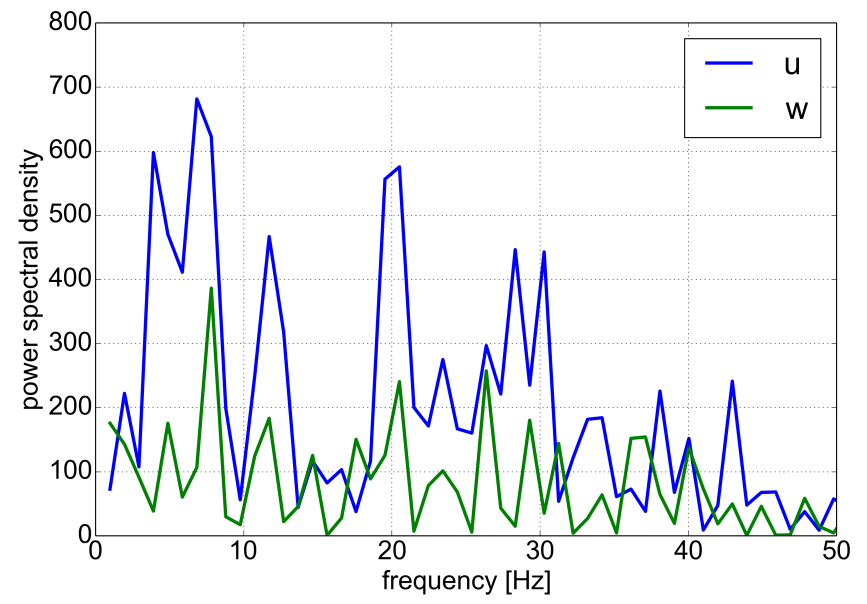

Figure 9: Streamwise and normal velocity ( $u$ and $w$ ) spectra above the aft deck.

To illustrate the sensitivity of the hangar wake to the overset boundaries near the ship, Fig. 10 depicts averaged flow fields for the linear and RBF overset approaches. The nearest overset boundary is located approximately 1.26 times the deck length downstream from the ship stern, near the edge of the contour 
plots in Figs. 10(b)-(d). On average, the boundary solutions differed on the order of $10^{-4}$ between methods. However, these variations were sufficient to modify the unsteady flow upstream of the boundary as differences are visible above the aft deck. Bold contour lines have been superimposed on Figs. 10(b)(d) to bracket the region between $0.7-0.8 V_{\infty}$ and highlight differences between the flow fields. To quantify the differences between the single and overset grid configurations, all solutions were collocated onto an identical Cartesian mesh. In comparison with the single-grid solution, average streamwise velocity errors were over $7 \%$ lower when applying TPS or W2 interpolation rather than trilinear interpolation. Average normal velocity errors were $15 \%$ and $35 \%$ lower than with linear interpolation when applying TPS and W2 functions, respectively. These differences translate into reduced errors in the deck surface solution.

As in the original SFS experiment [48, a recirculation region behind the hangar was observed. In addition, a secondary recirculation region between the step and the separation point has been reported in the literature, the extent of which tends to decrease with increasing Reynolds number [47. This feature was not clearly observed in the current results, likely due to the large Reynolds number. To estimate the locations at which the unsteady flow separates and reattaches on the deck, skin friction contours were extracted from the ship surface and contour lines at which the skin friction is zero were identified. The locations along the ship centerline at which the skin friction changes from positive to negative have been tabulated as the separation location (Table 8p. Similarly, the locations at which the skin friction changes from negative to positive have been tabulated as reattachment locations.

Under current flow conditions, the recirculation region spans over half the length of the flight deck. The flow separates from the deck at approximately half a hangar height away from the hangar, and reattaches at a distance of approximately 2.6 hangar heights along the deck. Digitized measurement data from Ref. 47 describe the separated flow in terms of skin friction and forward flow probability (FFP) distribution; when the flow has an equal probability of flowing forward or backward, that location is considered the separation point. 


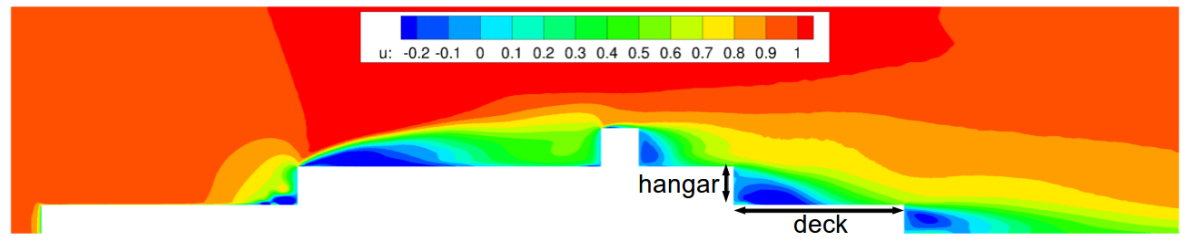

(a) Single Grid

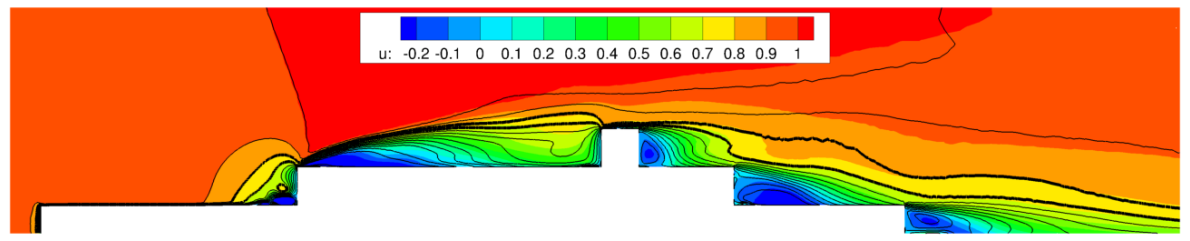

(b) Trilinear Overset (color contours)

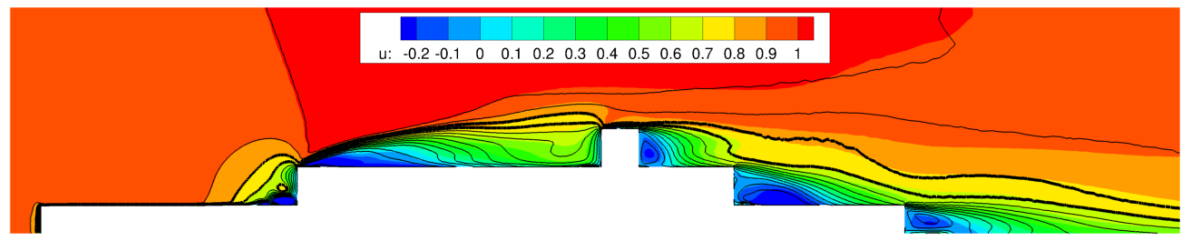

(c) Thin-Plate Splines Overset (color contours)

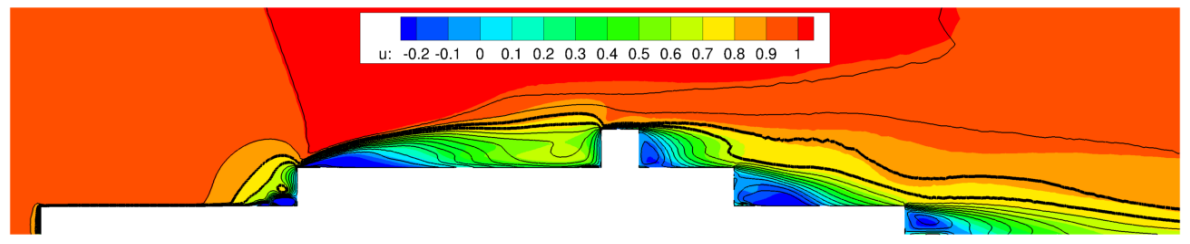

(d) Wendland $C^{2}$ Overset (color contours)

Figure 10: Averaged contours of streamwise velocity for single and overset grid configurations, viewed from the port side. In the overset cases, black line contours represent the single-grid solution. 
Table 8: Locations of separation and reattachment on the ship deck, normalized by hangar height.

\begin{tabular}{lccc}
\hline \hline Overset Method & Separation, $x_{s} / h$ & Reattachment, $x_{r} / h$ & $x_{s} / x_{r}$ \\
\hline Single Grid & 0.5247 & 2.5646 & 0.2046 \\
Trilinear Interpolation & 0.4763 & 2.6212 & 0.1817 \\
Thin-plate spline & 0.5175 & 2.6099 & 0.1983 \\
Wendland $C^{2}$ & 0.5171 & 2.6271 & 0.1968 \\
\hline \hline
\end{tabular}

The skin friction data do not indicate a strong dependence on Reynolds number and the calculated separation locations range between 0.1 and $0.25 x_{r}$. FFP data for the largest Reynolds number tested $\left(1.6 \times 10^{4}\right)$ locates the separation point near $0.20 x_{r}$ [47. Both methods predict an absolute separation location of $1.1-1.3 h$.

Experimental separation locations are approximately double the calculated separation locations (Table 8), owing to the finite length of the ship deck. In the SFS configuration, the length of the deck is $4.5 h$, while the experimental channel extended over $95 h$ [4] downstream of the step. Therefore the relative separation location is a more useful measure. In terms of the relative separation location, both calculations and experiment indicate that the flow separates from the deck at $0.20 x_{r}$, suggesting the validity of the single grid CFD model. The relative separation location calculated from the linearly interpolated overset simulation differs from the single-grid solution by $11 \%$. In comparison, TPS and W2 overset results are within 3 and $4 \%$ of the single-grid solution (Table 8 .

The ship airwake case was run in parallel on 256 processors. When applying RBF interpolation, the average size of the interpolation problem (in terms of the number of average donors) increases eightfold from 8 to 32 . In three dimensions, the number of donors is approximately $30 \%$ higher than the two-dimensional case, indicating increased cost. However, parallel solver operation reduces the number of fringe points per processor and as a result the added interpolation time was only $6 \%$ of the total solver cost per step (Table 9). 
Table 9: Cost in terms of averaged wallclock time per step for the test cases and methods presented.

\begin{tabular}{lcc}
\hline \hline Overset Method & Average cost/step [s] & $\%$ change \\
\hline Trilinear interpolation & 18.8474 & - \\
Thin-plate spline & 19.9706 & $6.0 \%$ \\
Wendland $C^{2}$ & 20.0179 & $6.2 \%$ \\
\hline \hline
\end{tabular}

\section{Conclusions}

The practical potential of an overset data transfer strategy based upon scattered data interpolation with radial basis functions (RBFs) has been demonstrated for two test cases. First, a simple vortex convection case was studied in detail; then experience from that study was applied to a complex vortexdominated problem. The methods outlined in this paper provide a framework for more general data transfers including configurations with multiple donor grids. Although not explored here, donor points may be arbitrarily sourced from any number of grids in the vicinity of a receptor point provided a donor grid suitability algorithm is implemented and/or grid preferences are explicitly specified for a given problem.

The observed solution accuracy in the presence of orphan points demonstrates the effectiveness of the RBF approach in both interpolation and extrapolation. Application of a cloud-based algorithm in place of linear interpolation is therefore recommended based on the following findings:

- RBF interpolation can significantly reduce overset interpolation error. In the vortex validation case, configurations both with and without orphan points converged with grid refinement and the RBF interpolation approaches consistently outperformed trilinear interpolation. Isolated overset errors were reduced by up to an order of magnitude with RBF interpolation in comparison with traditional trilinear basis functions.

- The RBF overset algorithm eliminates orphan points because clouds of 
non-fringe donor points can always be found in the vicinity of a receptor, enabling interpolation or extrapolation. This approach eliminates the need to perform averaging when suitable donors are lacking. In addition to reducing spatial errors, the $\mathrm{RBF}$ data transfer minimized transient conservation errors in the presence of orphans in the vortex validation case.

- Investigation of a three-dimensional turbulent separated flow demonstrated overset solution sensitivities to the choice of data transfer method. The RBF approach was able to preserve near-body flow physics through accurate data exchange between near- and far-field domains.

- The observed minimum cost increase for applying RBF interpolation was $2 \%$ and $6 \%$ in two and three dimensions respectively. Based on the vortex convection study, the computational cost appears to scale with the total number of fringe points squared. Since high node-count grids motivate parallel solver execution, the increase in fringe points per processor is naturally limited in practice, thus minimizing the increase in computational expense due to data transfer.

\section{Acknowledgments}

The computational work was supported in part by the Office of Naval Research (ONR) under the research grant "High-Accurate Physics-Based Wake Simulation Techniques" (Grant number N00014-10-C-0190), with guidance from the technical monitor Dr. Judah Milgram. Computing resources were provided by the Department of Defense High Performance Computing (HPC) Modernization Program.

This paper was prepared as an account of work sponsored by an agency of the United States (U.S.) Government. Neither the U.S. Government nor any agency thereof, nor any of their employees, makes any warranty, express or implied, or assumes any legal liability or responsibility for the accuracy, completeness, or usefulness of any information, apparatus, product, or process 
Reference herein to any specific commercial product, process, or service by trademark, manufacturer, or otherwise does not necessarily constitute or imply its endorsement, recommendation, or favoring by the U.S. Government or any agency thereof. The views and opinion expressed herein do not necessarily state or reflect those of the U.S. Government or any agency thereof.

\section{References}

[1] J. Steger, F. Dougherty, J. Benek, A Chimera Grid Scheme, American Society of Mechanical Engineers, Fluids Engineering Division 5 (1983) 5969.

[2] J. Benek, J. Steger, F. Dougherty, A Flexible Grid Embedding Technique with Application to the Euler Equations, in: 6th AIAA Computational Fluid Dynamics Conference, no. AIAA-83-1944, Danvers, Massachusetts, 1983.

[3] J. Abras, C. E. Lynch, M. Smith, Computational Fluid Dynamics-

[6] G. D. Power, J. A. Gudenkauf, J. Masters, J. A. Calahan, M. Aboulmouna, Integration of USM3D into the Store-Separation Process: Current Status 
and Future Direction, in: 47th AIAA Aerospace Sciences Meeting and Exhibit, AIAA-2009-339, Orlando, Florida, 2009.

[7] S. E. Rogers, N. E. Suhs, W. E. Dietz, PEGASUS 5: An Automated Preprocessor for Overset-Grid Computational Fluid Dynamics, AIAA Journal 41 (6) (2003) 1037-1045.

[8] J. Sitaraman, M. Floros, A. Wissink, M. Potsdam, Parallel Domain Connectivity Algorithm for Unsteady Flow, Journal of Computational Physics 229 (12) (2010) 4703-4723.

[9] R. W. Noack, D. A. Boger, R. F. Kunz, P. M. Garrica, Suggar++: An Improved General Overset Grid Assembly Capability, in: 19th AIAA Computational Fluid Dynamics Conference, AIAA-2009-3992, San Antonio, Texas, 2009.

[10] R. L. Meakin, On the Spatial and Temporal Accuracy of Overset Grid Methods for Moving Body Problems, in: 12th AIAA Applied Aerodynamics Conference, no. AIAA-94-1925, Colorado Springs, Colorado, 1994, 858-871.

[11] R. W. Noack, Resolution Appropriate Overset Grid Assembly for Structured and Unstructured Grids, in: 16th AIAA Computational Fluid Dynamics Conference, AIAA-2003-4123, Orlando, Florida, 2003.

[12] D. Gaitonde, M. Visbal, Padé-type Higher-Order Boundary Filters for the Navier-Stokes Equations, AIAA Journal 38 (11) (2000) 2103-2112.

[13] S. Sherer, J. Scott, High-Order Compact Finite-Difference Methods on General Overset Grids, Journal of Computational Physics 210 (2) (2005) 459-496.

710

[14] R. W. Noack, DiRTlib: A Library to Add an Overset Capability to Your Flow Solver, in: 17th AIAA Computational Fluid Dynamics Conference, no. AIAA-2005-5116, Toronto, Ontario, 2006. 
[15] S. E. Rogers, K. Roth, S. M. Nash, M. D. Baker, J. P. Slotnick, M. Whitlock, H. V. Cao, Advances in Overset CFD Processes Applied to Subsonic High-Lift Aircraft, in: 18th AIAA Applied Aerodynamics Conference, no. AIAA-2000-4216, Denver, Colorado, 2000.

[16] J. N. Abras, N. Hariharan, CFD Solver Comparison of Low Mach Flow over the ROBIN Fuselage, in: AIAA SciTech 52nd Aerospace Sciences Meeting and Exhibit, no. AIAA-2014-0752, National Harbor, Maryland, 2014.

[17] S. C. Platt, Parachute Extraction of a Generic Store from a C-130; a CFD Proof of Concept, Master's thesis, Air Force Institute of Technola ogy, Wright-Patterson Air Force Base, Ohio, http://www.dtic.mil/docs/ citations/ADA434226 (March 2005).

[18] R. Schaback, Creating Surfaces from Scattered Data Using Radial Basis Functions, in: Mathematical Methods for Curves and Surfaces, 1995, 477496.

[19] J.-C. Luo, Y. Leung, J. Zheng, J.-H. Ma, An Elliptical Basis Function Network for Classification of Remote Sensing Images, Journal of Geographical Systems 6 (2004) 219-236.

[20] H. Wendland, Scattered Data Approximation, Cambridge University Press, New York, NY, 2005.

[21] E. W. Quon, Data Transfer Strategies for Overset and Hybrid Computational Methods, Ph.D. thesis, Georgia Institute of Technology, Atlanta, Georgia (2014).

[22] R. Franke, Scattered Data Interpolation: Tests of Some Methods, Mathematics of Computations 38 (157) (1982) 181-200sch.

[23] M. J. Smith, D. H. Hodges, C. E. S. Cesnik, An Evaluation of Computational Algorithms to Interface Between CFD and CSD Methodologies, Tech. Rep. WL-TR-96-3055, Air Force Wright Laboratory (November 1995). 
[24] P. K. Lee, CFD/CSD Grid Interfacing of Three-Dimensional Surfaces by Inverse Isoparametric Mapping, Master's thesis, Massachusetts Institute of Technology, http://dspace.mit.edu/handle/1721.1/81568 (2001).

[25] D. Lazzaro, L. B. Montefusco, Radial Basis Functions for the Multivariate Interpolation of Large Scattered Data Sets, Journal of Computational and

[26] H. Wendland, Topics in Multivariate Approximation and Interpolation, Elsevier, 2005, Ch. Computational Aspects of Radial Basis Function Approximation, $231-256$.

[27] R. W. Shonkwiler, L. Lefton, An Introduction to Parallel and Vector Scientific Computing, Cambridge University Press, New York, NY, 2006.

[28] M. J. Smith, C. E. S. Cesnik, D. H. Hodges, Evaluation of Some Data Transfer Algorithms for Noncontiguous Meshes, Journal of Aerospace Engineering 13 (2) (2000) 52-58.

[29] M. J. Smith, D. H. Hodges, C. E. S. Cesnik, An Evaluation of Computational Algorithms Suitable for Fluid-Structure Interaction, AIAA Journal of Aircraft 37 (2) (2000) 282-294.

[30] A. de Boer, A. H. van Zuijlen, H. Bijl, Advanced Computational Methods in Science and Engineering, 2010, Ch. Radial Basis Functions for Interface Interpolation and Mesh Deformation, 143-178.

[31] T. Rendall, C. Allen, Efficient Mesh Motion Using Radial Basis Function with Data Reduction Algorithms, Journal of Computational Physics 228 (17) (2009) 6231-2649.

[32] W. Costin, C. Allen, Numerical Study of Radial Basis Function Interpolation for Data Transfer Across Discontinuous Mesh Interfaces, International Journal for Numerical Methods in Fluids 72 (2013) 1076-1095. doi:10.1002/fld.3778. 
[33] T. Rendall, C. Allen, Evaluation of RBFs for volume data interpolation in CFD, International Journal for Numerical Methods in Fluids 72 (2013) 748-769. doi:10.1002/fld.3766.

[34] A. Beckert, H. Wendland, Multivariate Interpolation for Fluid-StructureInteraction Problems Using Radial Basis Functions, Aerospace Science and Technology 5 (2) (2001) 125-134.

[35] W. K. Anderson, D. L. Bonhaus, An Implicit Upwind Algorithm for Computing Turbulent Flows on Unstructured Grids, Computers \& Fluids 23 (1) (1994) 1-21.

[36] V. N. Vatsa, M. H. Carpenter, Higher Order Temporal Schemes with Error Control for Unsteady Navier-Stokes Equations, in: 17th AIAA Computational Fluid Dynamics Conference, no. AIAA-2005-5245, Toronto, Canada, 2005 .

[37] R. Biedron, V. Vatsa, H. Atkins, Simulation of Unsteady Flows Using an Unstructured Navier-Stokes Solver on Moving and Stationary Grids, in: 23rd AIAA Applied Aerodynamics Conference, no. AIAA-2005-5093, Toronto, Ontario, 2005.

[38] R. W. Noack, D. A. Boger, R. F. Kunz, P. M. Carrica, Suggar++: An Improved General Overset Grid Assembly Capability, in: AIAA 19th Computational Fluid Dynamics Conference, no. AIAA-2009-3992, Austin, Texas, 2009.

[39] M. R. Visbal, D. V. Gaitonde, High-Order-Accurate Methods for Complex Unsteady Subsonic Flows, AIAA Journal 37 (10) (1999) 1231-1239.

[40] M. R. Visbal, D. V. Gaitonde, On the Use of Higher-Order Finite-Difference Schemes on Curvilinear and Deforming Meshes, Journal of Computational Physics 181 (2002) 155-185.

[41] S. Etienne, A. Garon, D. Pelletier, Code Verification for Unsteady Flow Simulation with High Order Time-Stepping Schemes, in: 47th AIAA 
Aerospace Sciences Meeting and Exhibit, no. AIAA-2009-0169, Orlando, Florida, 2009.

[42] W. Press, B. Flannery, S. Teukolsky, W. Vetterling, Numerical Recipes in FORTRAN: The Art of Scientific Computing, 2nd Edition, Cambridge University Press, New York, NY, 1992, Ch. 2.

[43] D. Roper, I. Owen, G. Padfield, S. Hodge, Integrating CFD and Piloted Simulation to Quantify Ship-Helicopter Operating Limits, The Aeronautical Journal 110 (1109) (2006) 419-428.

[44] E. Quon, M. J. Smith, N. Rosenfield, G. Whitehouse, Investigation of Ship Airwakes Using a Hybrid Computational Methodology, in: Proceedings of the 70th American Helicopter Society Annual Forum, Montréal, Canada, 2014.

[45] M. Smith, J. Cook, M. Sánchez-Rocha, R. Shenoy, S. Menon, Improved Prediction of Complex Rotorcraft Aerodynamics, in: Proceedings of the 69th American Helicopter Society Annual Forum, Phoenix, Arizona, 2013.

[46] A. Heenan, J. Morrison, Passive Control of Pressure Fluctuations Generated by Separated Flows, AIAA Journal 36 (6) (1998) 1014-1022.

[47] P. Spazzini, G. Iuso, M. Onorato, N. Zurlo, G. D. Cicca, Unsteady Behavior of Back-Facing Step Flow, Experiments in Fluids 30 (2001) 551-561.

[48] N. Wakefield, S. Newman, P. Wilson, Helicopter Flight Around a Ship's Superstructure, in: Institution of Mechanical Engineers Part G: Journal of Aerospace Engineering, Vol. 216, 2002, 13-28. 